\title{
Comparison of Tibialis Anterior Muscle Activity and Dorsiflexor Strength according to Toe Postures
}

\author{
In-cheol Jeon, PT, Ph.D ${ }^{1}$; Jun-hyeok Jang, PT, Ph.D \\ ${ }^{1}$ Department of Physical Therapy, Hoseo University, Asan, South Korea \\ ${ }^{2}$ Hill \& Heal Balance Center, Busan, South Korea
}

Background The strength of dorsiflexor muscle is frequently measured to evaluate the performance of ankle joint in the clinical setting. However, little research into comparison of tibialis anterior (TA) muscle activity and dorsiflexor strength according to toe postures during dorsiflexion has been reported.

Purpose The purpose was to compare TA muscle activity and dorsiflexor strength according to toe postures in sitting position. The TA muscle is very important in the ankle joint structures. The selective strengthening exercise for the TA muscle is essential to improve the performance of ankle joint.

Study design Study design Comparative, repeated measures design.

Methods Twenty five subjects was enrolled in this study. The TA muscle activity and dorsiflexor strength during dorsiflexion with toe flexion and toe extension were measured by using a surface EMG device and a hand held dynamometer. Paired $t$-test was used to confirm the significant difference between conditions. The level of statistical significance was set at $\alpha=0.05$.

Results The TA muscle activity and dorsiflexor strength were significantly different between dorsiflexion with toe flexion and with toe extension $(p<0.05)$. The dorsiflexor strength during dorsiflexion with toe flexion was significantly smaller compared to that with toe extension. In contrast, the TA muscle activity during dorsiflexion with toe flexion was significantly greater compared to that with toe extension $(p<0.05)$.

Conclusions The dorsiflexion with toe flexion can be more effective to selectively increase TA muscle activity compared to that with toe extension. The dorsiflexion with toe flexion for selective TA muscle strengthening can be recommended.

JMST

2020; 4(1): 13-17 Published Online Jun 30, 2020 pISSN 2635-8573 eISSN 2635-858

Article History Received 30 Apr 2020 Revised 18 May 2020 (1st) Revised 21 May 2020 (2nd) Accepted 27 May 2020

\section{CONTACT}

jangpt@hanmail.net Jun-hyeok Jang Hill \& Heal Balance Center, Busan, South Korea

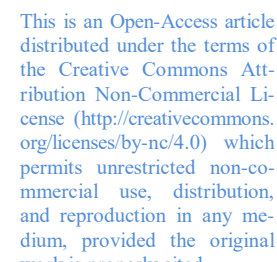
work is properly cited.

Key words Muscle activity; Strength; Tibialis anterior; Toe postures.

\section{INTRODUCTION}

The tibialis anterior (TA) muscle is an essential component in ankle and knee joint structure. ${ }^{1}$ The TA muscle was in function as a dorsiflexor and as a dynamic stabilizer of ankle joint during runnning and jumping. ${ }^{1,2}$ In addition, the TA muscle have a role of maintaining the axis of ankle joint during dorsiflexion. ${ }^{1,2}$ The insufficient TA muscle strength compared to extensor hallucis longus (EHL), and extensor digitorum longus (EDL) can result in the frequent and excessive compensations of ankle joint during dorsiflexion causing ankle instability and hammer toe. ${ }^{3}$ Therefore, the selective strengthening exercise of TA muscle is one of the primary element in effective ankle rehabilitation. ${ }^{1}$

A dominant EHL and EDL muscle compared to TA can influence on ankle instability and result in alterations in the movement strategy of ankle joint which result in toe deformities. ${ }^{4-6}$ Dominant and compensated extension of the 
metatarsophalangeal joint (MTPJ) and flexion of the proximal interphalangeal (IP) joint can cause hammer toes, and the abduction of the MTPJ can evoke hallux valgus. ${ }^{1,3,7}$ These impairments of ankle and foot structures may be influenced by muscle imbalance among the TA, EHL and EDL muscle.

As a pure dorsiflexor, TA muscle is needed to be stronger compared to other synergistic dorsiflexors such as EHL and EDL. ${ }^{8,9}$ The performance of dorsiflexion in ankle movement can be influenced by $55 \%$ of TA, $30 \%$ of EDL and $15 \%$ of EHL. ${ }^{10}$ Most studies have focused on the types, intensity and times of ankle dorsiflexor strengthening exercises. ${ }^{11}$ However, the study has not yet investigated comparison of TA muscle activity and dorsiflexor strength according to toe postures during dorsiflexion.

The extrinsic toe extensors could be effectively inhibited by toe flexion during ankle dorsiflexion. ${ }^{1}$ The active toe flexion may be helpful to increase TA muscle activity and reduce EHL and EDL activity during dorsiflexion in subjects with EHL and EDL dominance. ${ }^{2}$ However, no study is investigated comparison of TA muscle activity and dorsiflexor strength during dorsiflexion according to toe flexion and extension posture. Therefore, the aim of this study was to compare TA muscle activity and dorsiflexor strength during dorsiflexion with toe flexion and extension. The research hypothesis was that TA muscle activity during dorsiflexion with toe flexion would be greater compared to that with toe extension. In contrast, the dorsiflexor strength during dorsiflexion with toe flexion would be smaller compared to that with toe extension.

\section{METHODS}

\section{Participants}

G*Power software was used (ver. 3.1.2; Franz Faul, University of Kiel, Kiel, Germany) for pilot study of ten participants. The sample size was calculated with a power (0.80), an alpha level (0.05), and an effect size (1.29). The sample size was required at least fourteen participants. Twenty five subjects were recruited for this study. The general characteristics of the subjects are summarized in Table 1. Inclusion criteria included: (1) normal ankle joint range of motion, (2) no dysfunctions of musculoskeletal and

Table 1. General characteristics of the subjects

$(\mathrm{N}=25)$

\begin{tabular}{cc}
\hline Variable & Mean \pm standard deviation \\
\hline Age $(\mathrm{yr})$ & $25.2 \pm 4.2$ \\
Height $(\mathrm{cm})$ & $174.1 \pm 5.5$ \\
Body mass $(\mathrm{kg})$ & $63.2 \pm 3.4$ \\
Gender & 14 males, 11 females \\
\hline
\end{tabular}

neurological region, (3) no surgery of history of ankle joint. Exclusion criteria were if they had 1) impaired sensation; 2) previous neuromuscular disorder; 3 ) previous surgery to the back, hip, knee, ankle; 4) hip, knee, or ankle pathologies (within the past 2 years), 5) fracture to the ankle; and 6) pain in any region of the body while testing. The experimental protocols were explained in detail to subjects, and all subjects provided written informed consent with the ethical principles of the Declaration of Helsinki.

\section{Dorsiflexor strength measurement}

The maximal strength of the dorsiflexor was measured in sitting position with one knee flexion $90^{\circ}$ which was tested using the dominant side. The strength measurement of the dominant side was performed as a tested side. To measure the strength of the dorsiflexor muscle, the break test was performed in sitting position. The upper body of the subjects leaned back comfortably with both arms supporting the upper body. A PowerTrack II $^{\mathrm{TM}}$ Commander dynamometer (JTECH Medical, Salt Lake City, USA) was used as a hand-held dynamometer to measure dorsiflexor muscle strength. The force was measured for all of the subjects by the dynamometer $(0.1 \mathrm{~N}$ unit). The dynamometer was placed over the dorsal surface of the foot at $8 \mathrm{~cm}$ distance from the lateral malleolus and in the direction of plantar flexion of the ankle joint (Figure 1). To standardize the placement of the dynamometer between subjects, the placement of dynamometer was marked over the dorsal surface of the foot at the same region. The subjects were required to maintain ankle dorsiflexion with all toes extension and ankle neutral position for 5 seconds while downward pressure was applied by the examiner. And then, subjects were required to maintain ankle dorsiflexion with all toes flexion and ankle neutral position for 5 seconds while downward pressure was

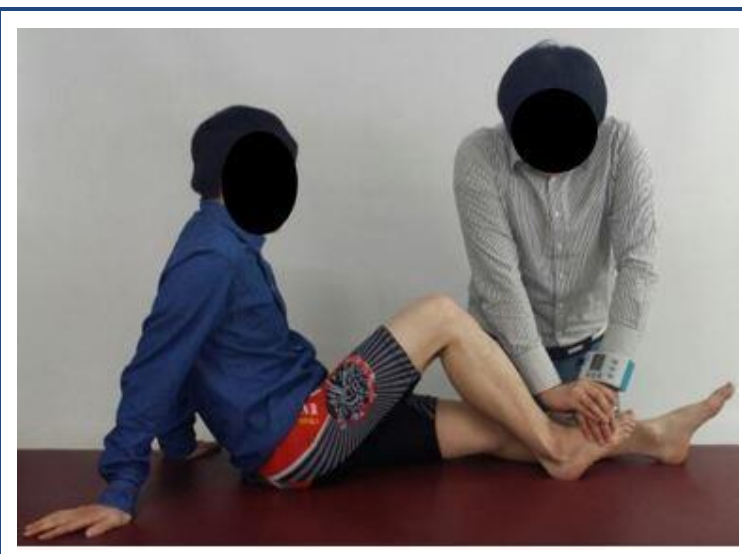

Figure 1. Measurement of strength of tibialis anterior according to toe postures. 
applied by the examiner. ${ }^{12}$ The order of task according to toe postures was randomized. The measurements were performed 3 times with 2 minutes resting time between trials which was for preventing the fatigue. ${ }^{13}$ The trial data values were averaged.

\section{Electromyography recording and data analysis}

Surface electromyography (EMG) feedback was provided by a wireless telemetry system (TeleMyo 2400T, Noraxon, Scottsdale, AZ, USA), and MyoResearch Master Edition 1.06 XP (Noraxon, Scottsdale, AZ, USA) was used to analyze the EMG signals. The EMG signals were amplified using a 1,000 gain factor, analogue to digital converted and saved with 12 bit resolution and 1,000 Hz/channel. A digital band-pass filter (Lancosh FIR, 20-450 Hz) was used to remove movement artifacts, and a notch filter was present to reject any values above $60 \mathrm{~Hz}$. The sampling rate was set at 1,024 Hz. The EMG signals were processed as rootmean-square (RMS) data with a moving window of $50 \mathrm{~ms}$. While participants maintained the dominant ankle dorsiflexion, EMG signals were recorded for $5 \mathrm{~s}$. EMG signals from 2 to $4 \mathrm{~s}$ were used for the analysis. The middle $3 \mathrm{~s}$ during the isometric phase of each exercise was used to prevent possible confounding effects due to the start and stop of the exercise. ${ }^{14,15}$ To minimize skin resistance, the skin over the TA muscle was prepared by cleansing with isopropyl alcohol before electrode placement; then, disposable $\mathrm{Ag} / \mathrm{AgCl}$ surface electrodes were fixed on the appropriate sites. ${ }^{16}$ Furthermore, the electrodes are placed $3 \mathrm{~cm}$ apart and parallel to the muscle fibers of TA over the muscle belly. ${ }^{16}$ All the tested sides were on the right ankle, which for the purposes of the study was classified as the dominant limb.

\section{Normalization}

The manual muscle testing positions for normalization using a maximum voluntary isometric contraction (MVIC) of TA was trained according to the guidelines of Kendall et al. ${ }^{17}$ The first and last second of the EMG signals of the MVIC tasks were discarded, and the $3 \mathrm{~s}$ of remaining data were used for analysis. The mean value of the three MVIC trials was calculated and all EMG signals were expressed as percentages of the MVIC (\%MVIC).

\section{Statistical analysis}

The data are expressed as the means \pm standard deviations. Kolmogorov-Smirnov test was employed to ensure the normal distribution for the data collected through the measurements. Paired $t$-test was employed for the difference between TA muscle activity and dorsiflexor strength according to toe postures (dorsiflexion with toe flexion vs. with toe extension). $p$-values $<0.05$ were deemed to indicate statistical significance. The statistical package for the Social Sciences for Windows version 18.0 (SPSS, Inc., Chicago, IL, USA) was used for statistical analysis.

\section{RESULTS}

The TA muscle activity and dorsiflexor strength were significantly different between toe postures (dorsiflexion with toe flexion vs. with toe extension) ( $p<0.05$; Table 2 and 3). The TA muscle activity during dorsiflexion with toe flexion was significantly greater compared to that with toe extension $(p<0.05$; Table 2$)$. In contrast, the dorsiflexor strength during dorsiflexion with toe flexion was significantly smaller compared to that with toe extension $(p<0.05$; Table 3).

Table 2 . The difference in TA muscle activity between conditions

\begin{tabular}{|c|c|c|c|c|}
\hline \multirow{2}{*}{ Variables } & \multicolumn{2}{|c|}{ Mean $\pm \mathrm{SD}^{\mathrm{a}}(\%)$} & \multirow{2}{*}{$t$ value } & \multirow{2}{*}{$p$ value } \\
\hline & Dorsiflexion with toe flexion & Dorsiflexion with toe extension & & \\
\hline$\% \mathrm{MVIC}$ & $69.01 \pm 15.46$ & $54.53 \pm 26.34$ & 8.28 & $<0.05^{*}$ \\
\hline
\end{tabular}

${ }^{*} p<0.05$, by paired $t$-test.

${ }^{\mathrm{a}} \mathrm{SD}$, standard deviation.

Table 3. The difference in dorsiflexor strength between conditions

\begin{tabular}{|c|c|c|c|c|}
\hline \multirow[b]{2}{*}{ Variables } & \multicolumn{2}{|c|}{$\operatorname{Mean} \pm \mathrm{SD}^{\mathrm{a}}(\mathrm{kg})$} & \multirow[b]{2}{*}{$t$ value } & \multirow[b]{2}{*}{$p$ value } \\
\hline & $\begin{array}{l}\text { Dorsiflexion with toe flexion } \\
\text { toe flexion }\end{array}$ & $\begin{array}{l}\text { Dorsiflexion with toe flexion } \\
\text { toe extension }\end{array}$ & & \\
\hline Strength & $26.82 \pm 5.08$ & $35.27 \pm 12.41$ & -6.27 & $<0.05^{*}$ \\
\hline
\end{tabular}

${ }^{*} p<0.05$, by paired $t$-test.

${ }^{\text {a }} \mathrm{SD}$, standard deviation. 


\section{DISCUSSION}

The purpose of this study was to compare TA muscle activity and dorsiflexor strength according to toe postures. The result of this study showed that TA muscle activity during dorsiflexion with toe flexion was significantly greater compared to that with toe extension $(p<0.05 ; 26.55 \%$ difference). In contrast, the dorsiflexor strength with toe flexion was significantly smaller compared to that with toe extension ( $p<0.05 ;-31.51 \%$ difference). In this study's result reported that even though the dorsiflexor strength with toe flexion was smaller compared to that with toe extension, the TA muscle activity during dorsiflexion with toe flexion was greater that with toe extension.

There was some explanation to explain these findings. First, the dorsiflexor strength can be elicited by the various muscles such as TA, EHL and EDL as a dorsiflexion synergists. Therefore, the selective TA muscle strengthening as a prime mover of dorsiflexion can be effective without coactivation of EHL and EDL as accessory muscles. ${ }^{1,2}$ The TA muscle can be principally performed as a dorsiflexor in the ankle joint. Therefore, the muscle balance among the TA, EDL and EHL muscles was needed to consider the toe postures for the activation of TA and reduction of EDL and EHL during dorsiflexion. ${ }^{1,2}$

Second, the greater dorsiflexor strength can be occurred with dorsiflexion with toe extension because the TA, EHL and EDL muscles were co-activated. In contrast, the smaller dorsiflexor strength can be occurred with dorsiflexion with toe flexion because the TA muscle was activated, whereas the EHL and EDL muscle were inhibited. Indeed, volitional active muscle contractions could produce the improvement of muscle balance through the reciprocal inhibition mechanism. ${ }^{1,2,4}$ While the EHL and EDL muscle were stretched due to active toe flexion during dorsiflexion, volitionally induced inhibition could contributed to the decrease of the EHL and EDL muscle contraction. ${ }^{18,19}$ These components were significantly effective for optimal muscle balance during dorsiflexion especially in subjects with the EHL and EDL muscle dominance.

Third, the more tension was generated with the shortened position of the EHL and EDL muscles while toe extension was performed as a length-tension relationship. ${ }^{1,2,20}$ The observed increase of the TA muscle activity during dorsiflexion with toe flexion was occurred to generate the performance with the inhibition of the EHL and EDL muscles. Since these muscles become lengthened during dorsiflexion with toe flexion, the EHL and EDL muscle may be inhibited with less tension because of toe flexion during dorsiflexion. ${ }^{1,2}$ Whereas the EHL and EDL muscles can attribute to the dorsiflexor strength with more tension during dorsiflexion with toe extension. These implied that toe flexion and extension posture during dorsiflexion can influence on selective muscle activation among the TA, EHL and EDL muscles.

The current study had several limitations. First, the results cannot be generalized to the subjects with dorsiflexor weakness. Further study is needed to investigate subjects with dorsiflexor weakness and other impairments of ankle joint and foot such as hammer toe and hallux valgus. Second, the EHL and EDL muscles activity was not investigated during dorsiflexion. Further study is required to attach the muscle activity of the EHL and EDL muscles during dorsiflexion. Third, the results cannot be generalized to the other ankle positions. Further study is needed to investigate TA muscle activity according various ankle positions such as inversion and eversion. Fourth, the strength of ankle dorsiflexors was not measured by using a fixing frame for a dynamometer.

\section{Key Points}

Question Can the strength of dorsiflexor muscle be different between dorsiflexion with toe flexion and toe extension?

Findings The findings of this study reported that the tibialis anterior muscle activity during dorsiflexion with toe flexion can be greater compared to that with toe extension.

Meaning The intervention for strength of dorsiflexor muscle should be different especially in toe postures. The dorsiflexion with toe flexion for strengthening of selective tibialis anterior muscle can be recommended.

\section{Article information}

Conflict of Interest Disclosures: None.

Funding/Support: This work was supported by the National Research Foundation of Korea (NRF) grant funded by the Korea government (MSIT) (No. 2017R1 C1B5076172).

Acknowledgment: None.

Ethic Approval: This study was approved by the Hoseo University Institutional Review Board.

\section{REFERENCES}

1. Sahrmann SA. Diagnosis and treatment of movement impairment syndromes. St Louis: Mosby; 2002.

2. Kisner C, Colby LA. Therapeutic exercise: foundations and techniques. Philadelphia: FA Davis Company; 2012.

3. Kwon OY, Tuttle LJ, Johnson JE, Mueller MJ. Muscle 
imbalance and reduced ankle joint motion in people with hammer toe deformity. Clin Biomech (Bristol, Avon). 2009;24(8):670-675.

4. Page P, Frank C, Lardner R. Assessment and treatment of muscle imbalance: the Janda approach. Champaign: Human Kinetics; 2010.

5. Scheck M. Etiology of acquired hammertoe deformity. Clin Orthop Relat Res. 1977;123:63-69.

6. Hansen ST. Functional reconstruction of the foot and ankle. Philadelphia: Lippincott Williams \& Wilkins; 2000.

7. Dhukaram V, Hossain S, Sampath J, Barrie JL. Correction of hammer toe with an extended release of the metatarsophalangeal joint. J Bone Joint Surg Br. 2002; 84(7):986-990.

8. Marciniak C. Fibular (peroneal) neuropathy: electrodiagnostic features and clinical correlates. Phys Med Rehabil Clin N Am. 2013;24(1):121-137.

9. Hislop H, Avers D, Brown M. Daniels and Worthingham's muscle testing: techniques of manual examination and performance testing. Philadelphia: Elsevier; 2013.

10. Franck WM, Olk A, Hennig FF. Combined rupture of the tibialis anterior and the extensor hallucis longus tendons-functional reconstruction. Arch Orthop Trauma Surg. 2005;125(4):277-280.

11. Wernbom M, Augustsson J, Thomeé R. The influence of frequency, intensity, volume and mode of strength training on whole muscle cross-sectional area in humans. Sports Med. 2007;37(3):225-264.

12. Docherty CL, Moore JH, Arnold BL. Effects of strength training on strength development and joint position sense in functionally unstable ankles. $J$ Athl Train. 1998;33(4):310-314.

13. O'Shea S, Grafton K. The intra and inter-rater reliability of a modified weight-bearing lunge measure of ankle dorsiflexion. Man Ther. 2013;18(3):264-268.

14. Soderberg GL Knutson LM. A guide for use and interpretation of kinesiologic electromyographic data. Phys Ther. 2000;80(5):485-498.

15. Ayotte NW, Stetts DM, Keenan G, Greenway EH. Electromyographical analysis of selected lower extremity muscles during 5 unilateral weight-bearing exercises. $J$ Orthop Sports Phys Ther. 2007;37(2):48-55.

16. Criswell E. Cram's introduction to surface electromyography. Sudbury: Jones \& Bartlett Publishers; 2010.

17. Kendall FP, McCreary EK, Provance PG, Rodgers MM, Romani WA. Muscles testing and function, with posture and pain. Baltimore: Lippincott Williams \& Wilkins; 2005.

18. Condon SM, Hutton RS. Soleus muscle electromyographic activity and ankle dorsiflexion range of motion during four stretching procedures. Phys Ther. 1987; 67(1):24-30.

19. Sady SP, Wortman M, Blanke D. Flexibility training: ballistic, static or proprioceptive neuromuscular facilitation?. Arch Phys Med Rehabil. 1982;63(6):261-263.

20. Jeon IC, Jang JH. Comparison of prone hip extension exercise and prone hip extension exercise after iliopsoas stretching on lumbopelvic control and gluteus maximus activity in subjects with short iliopsoas. J Musculoskelet Sci Technol. 2017;1(1):19-25. 